\title{
Anomalous Superconductivity and Antiferromagnetism in the $\mathrm{U}(\mathbf{P t}, \mathrm{Pd})_{3}$ System
}

\author{
J.J.M. FransE \\ Universiteit van Amsterdam, Spui 21, Amsterdam, The Netherlands
}

(Received September 20, 2001)

\begin{abstract}
A review is presented of the anomalous magnetic and superconducting properties of the heavy-fermion compound $\mathrm{UPt}_{3}$ that has been a subject of extensive study during the past twenty years. The normal-state and superconducting properties of the compound were evaluated from magnetic, transport, and thermodynamic studies. In addition, neutron-diffraction and muon spin relaxation experiments were performed in the normal and superconducting states. The compound is characterized by a multiple superconducting phase diagram and by the coexistence of superconductivity and short-range antiferromagnetic interactions with quasi-static moments of the order of $10^{-2} \mu_{\mathrm{B}} / \mathrm{U}$-atom. Upon substituting $\mathrm{Pt}$ by $\mathrm{Pd}$, the short-range magnetic interactions give way to long-range antiferromagnetic order with a maximal ordering temperature of $6 \mathrm{~K}$ for the 5 at.\% Pd alloy and a magnetic moment of $0.6 \mu_{\mathrm{B}} / \mathrm{U}$-atom. Superconductivity and long-range antiferromagnetic order are in competition resulting in a critical composition of 0.6 at.\% Pd. At this concentration, non-Fermi liquid behaviour is observed.
\end{abstract}

PACS numbers: 71.27.+a, 74.70.Tx, 75.30.Mb

\section{Introduction}

It is about twenty years from now that a search for the electronic and magnetic properties of the $\mathrm{U}-\mathrm{Pt}$ compounds has led to the discovery of $\mathrm{UPt}_{3}$ as a heavy-fermion system due to its anomalously large low-temperature electronic specific heat coefficient $[1,2]$. And although more striking magnetic properties were found in orthorhombic UPt [3,4], it was clear from the very beginning that the magnetism of the hexagonal compound $\mathrm{UPt}_{3}$ is interesting as well: at around 
$18 \mathrm{~K}$ a pronounced maximum was found in the susceptibility perpendicular to the hexagonal axis and at lower temperatures, a metamagnetic transition was observed in high magnetic field studies [1, 2]. This all was in contrast to $\mathrm{UPt}_{2}$ and $\mathbf{U P t}_{5}$ in which compounds no specific features in the thermal and magnetic properties were reported. The magnetic order in UPt turned out to be rather complex and sometimes confusing with an intermixing of magnetic and structural phase transitions and with complex hysteretic phenomena in temperature and high pressure cycles.

Returning now to the magnetic properties of $\mathrm{UPt}_{3}$, one of the more interesting phenomena was found to be the metamagnetic transition at $20 \mathrm{~T}$ that was studied in great detail in susceptibility [5, 6], magnetostriction [7], Hall-resistance [8], magnetoresistance [9], sound-velocity [10] and specific-heat measurements [11, 12]. In all experiments the metamagnetic transition shows up as a well-defined phenomenon without giving, however, a real clue to the magnetic state below and above the metamagnetic field. High-pressure experiments show a shift of the metamagnetic transition to higher field values at increased pressures, whereas partly substitution of isoelectronic Pd brings the transition to lower field values. Since the effect of Pd substitution is to reduce the elementary cell volume these results were considered to be intriguing. Anyhow, from the specific-heat results it became clear that after reaching maximum values for $c / T$ at the metamagnetic transition field, the heavy-fermion state becomes suppressed at higher field values.

Some of the experimental results are displayed in Fig. 1 for the magnetization, in Fig. 2 for the specific heat and in Fig. 3 for the magnetoresistance at different pressures. As far as the magnetic properties are concerned, a maximum in the perpendicular susceptibility near $18 \mathrm{~K}$ and the anisotropy in the susceptibility for field directions perpendicular and parallel to the hexagonal axis reveal some resemblance with the compound $\mathrm{PrNi}_{5}$ and could, in principle, be explained by

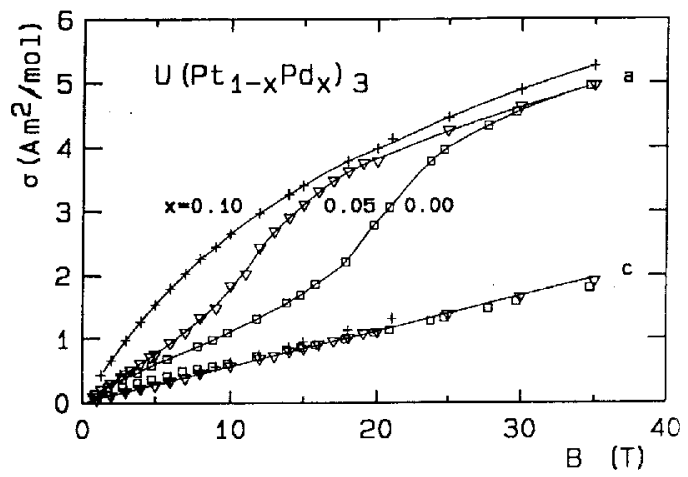

Fig. 1. The magnetization of hexagonal $\mathrm{UPt}_{3}$ along different crystallographic directions ( $a$ - and $c$-axis) at $T=4.2 \mathrm{~K}$; results for $\mathrm{U}\left(\mathrm{Pt}_{0.95} \mathrm{Pd}_{0.05}\right)_{3}$ and $\mathrm{U}\left(\mathrm{Pt}_{0.90} \mathrm{Pd}_{0.10}\right)_{3}$ are shown as well; the data show a shift of the metamagnetic transition along the $a$-axis from $20 \mathrm{~T}$ for $\mathrm{UPt}_{3}$ to zero tesla for the $\mathrm{U}\left(\mathrm{Pt}_{0.90} \mathrm{Pd}_{0.10}\right)_{3}$ compound; data from Ref. [6]. 

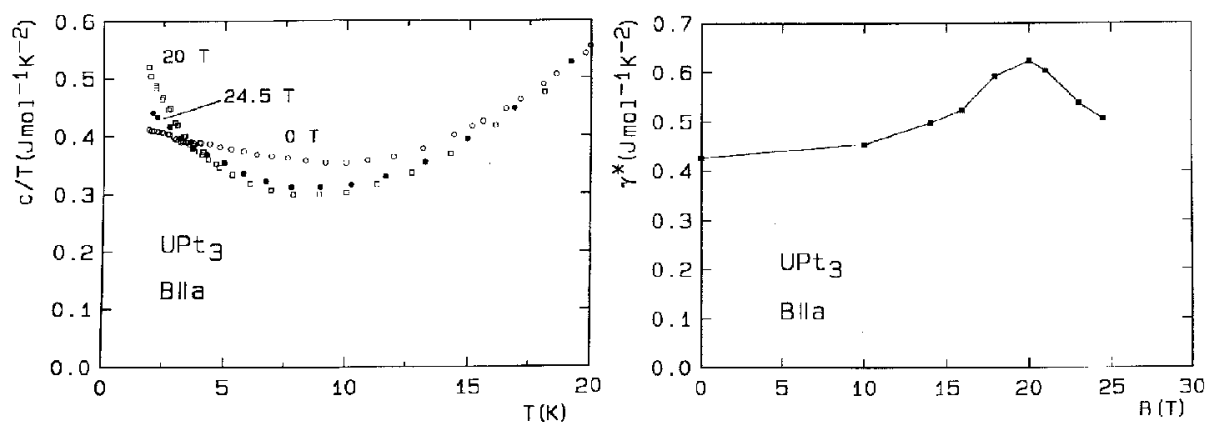

Fig. 2. The specific heat of single-crystalline UPt $\mathrm{U}_{3}$ in a plot of $c / T$ vs. $T$ at different field strengths along the $a$-axis (left part); values for the electronic specific-heat coefficient $\gamma^{*}$ as a function of magnetic field applied along the $a$-axis (right part); data from Ref. [12].

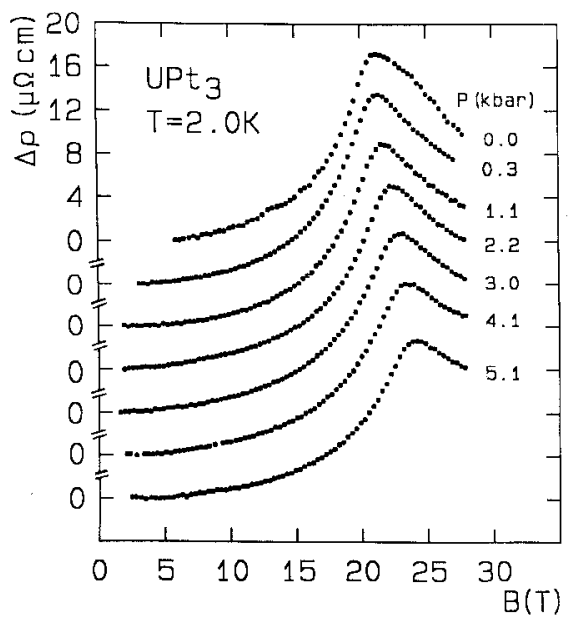

Fig. 3. High-field magnetoresistance of $\operatorname{UPt}_{3}(B\|i\| a)$ at $T=2.0 \mathrm{~K}$ under hydrostatic pressures up to $5.1 \mathrm{kbar}$; the metamagnetic field increases at a constant rate of $0.60 \mathrm{~T} / \mathrm{kbar}$; data from Ref. [9].

crystal field effects, assuming an identical electronic configuration for $\mathrm{U}$ in $\mathrm{UPt}_{3}$ as for $\operatorname{Pr}$ in $\mathrm{PrNi}_{5}$ [13]. However, the combination of the huge $c / T$ values at lower temperatures with the absence in thermodynamic and magnetic measurements of any tendency for incipient magnetic order (the very weak moments discovered in neutron-diffraction experiments will be discussed later on) ruled that possibility out, notwithstanding the large similarities in the thermal expansion, $\alpha(T)$, between the two compounds along the different crystallographic directions. But here again, the low-temperature behaviour of $\alpha / T$ on its turn showed anomalously large low-temperature values with Grüneisen parameter values about two orders of 
magnitude larger than in normal metals [14]. It proves that volume anomalies connected to the heavy-fermion state are even more pronounced than the anomalous low-temperature energy spectrum of the heavy-fermion state is. In the early days of the heavy-fermion research, efforts have been made in studying the implications of the large values for the electronic Grüneisen parameter, including a so-called Grüneisen analysis in which the different contributions to specific heat and thermal expansion were sorted out [15]. The heavy-fermion contribution to both quantities has been separated in this way from the remaining terms in an analysis that has been extended up to $300 \mathrm{~K}$. The entropy associated with the heavy-fermion contribution to the specific heat saturates above $100 \mathrm{~K}$ and reaches a value of, approximately, $R \ln 2$ near room temperature, resembling a spin- $1 / 2$ Kondo contribution although clear differences exist in the maximum value and in the peak width of the entropy as a function of temperature, see Fig. 4 . The relative volume change associated with the heavy-fermion state equals $0.9 \times 10^{-3}$ which volume reduction can be associated via the magnetovolume parameter with a reduction of the magnetic moment per uranium atom of about $2 \mu_{\mathrm{B}}$ at realising the heavy-fermion state. Apart from the conclusion that large values for the Grüneisen parameter have to be expected for compounds in which Kondo phenomena play an important role, further insights about, for instance, the microscopic nature of the heavy-fermion state or the presence of crystal-field contributions, did not emerge from this analysis. And although the resistivity of $\mathrm{UPt}_{3}$ itself exhibits a quadratic temperature

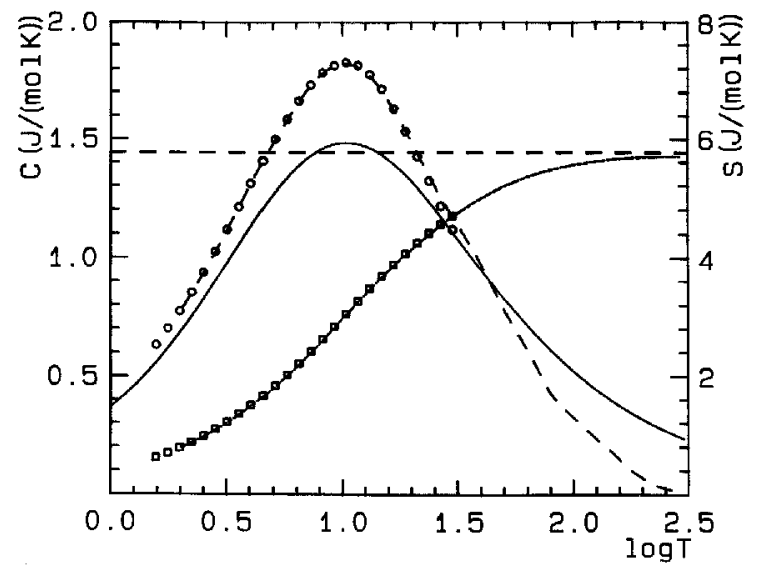

Fig. 4. The electronic contribution to the specific heat of $\mathrm{UPt}_{3}$ obtained from a Grüneisen analysis; the circles and the dashed line represent a low- and high-temperature analysis, respectively; the full line without symbols represents a theoretical $S=1 / 2$ single-ion Kondo contribution with $T_{\mathrm{K}}=15.5 \mathrm{~K}$; the squares represent the entropy and the full line through the squares, the high-temperature result; at high temperatures the entropy of $\mathrm{UPt}_{3}$ approaches the $R \ln 2$ level; data from Ref. [15]. 


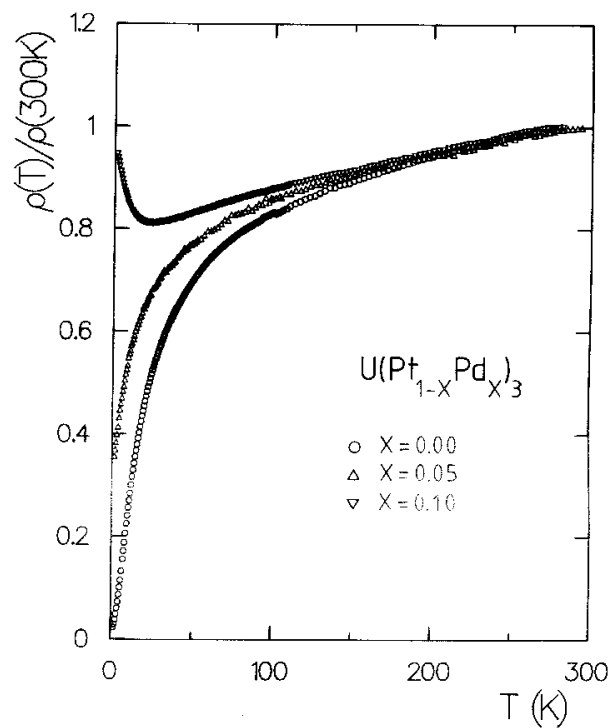

Fig. 5. Resistivity of $\mathrm{U}\left(\mathrm{Pt}_{1-x} \mathrm{Pd}_{x}\right)_{3}$ whiskers measured along the hexagonal axis as a function of temperature; the data have been normalised to 1 at $300 \mathrm{~K}$; note the change from Fermi-liquid to Kondo behaviour for $x$-values between 0.00 and 0.10 ; data from Ref. [16].

dependence at the lowest temperatures and a continuous rise with increasing temperature pointing to Fermi-liquid behaviour below $1 \mathrm{~K}$, Kondo-like phenomena in the resistivity could easily be evoked by substitution of platina by palladium [16], see the 10 at.\% Pd curve in Fig. 5. Part of these substitution studies were carried out on $\mathrm{U}(\mathrm{Pt}, \mathrm{Pd})_{3}$ whiskers that easily grow out of the melt under favourable conditions [17]. The whiskers have their long axis along the hexagonal direction with lengths up to a few $\mathrm{mm}$. The other dimensions are of micrometer scale. For the 5 at.\% Pd compound in Fig. 5, long-range antiferromagnetic order has been found below $6 \mathrm{~K}$. In the resistivity curve this phase transition is visible as a weak Cr-type anomaly around $6 \mathrm{~K}$. Continuous efforts have been made in the subsequent years to further improve the sample quality which led to room temperature over zero temperature resistance ratio (RRR) values up to 500 to 1000 for the best single-crystalline samples.

Soon after the first reports on the anomalous electronic properties of $\mathrm{UPt}_{3}$ at low temperatures, superconductivity was reported [18]. It made $\mathrm{UPt}_{3}$ the third heavy-fermion superconductor after $\mathrm{CeCu}_{2} \mathrm{Si}_{2}[19]$ and $\mathrm{UBe}_{13}$ [20]. From specific-heat studies near the superconducting transition it was clear that the heavy-mass electrons indeed take part in the superconducting condensate: the value for $\Delta c / \gamma T_{\mathrm{c}}$ is of the order of the BCS value of 1.43 , with an extrapolated value for $\gamma$ from temperatures above $T_{c}$ down to zero temperature of about $430 \mathrm{~mJ} /\left(\mathrm{mol} \mathrm{K}^{2}\right)$ and 
values for $T_{\mathrm{c}}$ and $\Delta c$ of $0.5 \mathrm{~K}$ and about $200 \mathrm{~mJ} /(\mathrm{mol} \mathrm{K})$.

As a result of the improved sample quality, new phenomena have been discovered: a structure in the transition to the superconducting state [21], see also Fig. 6 [22], and quantum oscillations in the magnetization [23] after unsuccesful first attempts to observe these phenomena on samples of less quality. This structure in the specific heat at the superconducting transition provided a natural explanation for the kink in the $B_{\mathrm{c} 2}(T)$ curve that was observed in earlier experiments [24] and turned out to be the proof that more than one superconducting phase is present in $\mathrm{UPt}_{3}$. It was found that the zero-field splitting of the superconducting transition is reduced in applied magnetic fields along the different crystallographic directions although the phenomenon is most resistant against fields along the $c$-direction. Further studies, among them thermal expansion measurements $[25,26]$, could show that in fact there are three distinguished phases in the superconducting state denoted by $A, B$, and $C$, see Fig. 7. The phase $C$ is a phase that can only be observed in applied magnetic fields. Subsequent high-

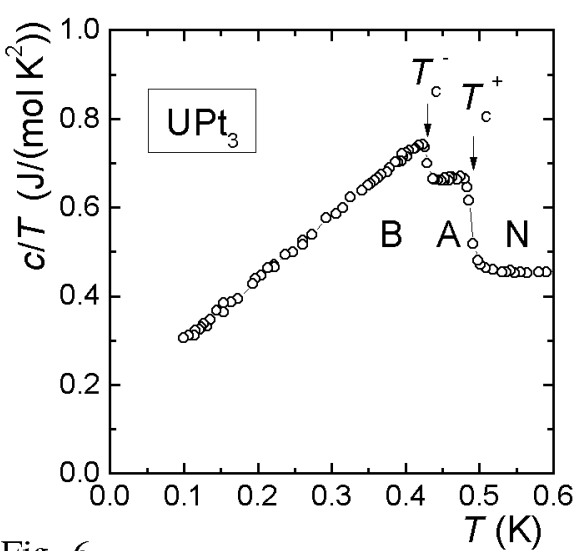

Fig. 6

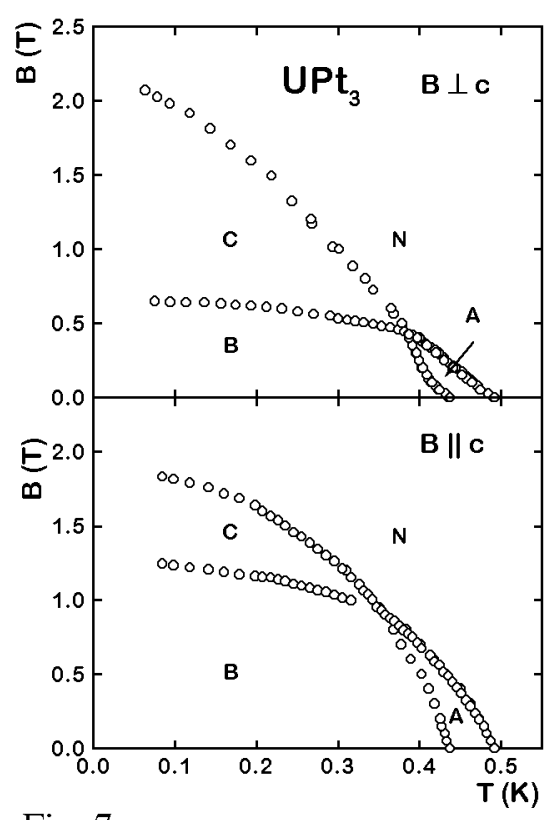

Fig. 7

Fig. 6. The transition to the superconducting state as observed in specific-heat measurements; plotted is $c / T$ vs. $T$; two superconducting transitions can be distinguished at $T_{c}^{+}$and $T_{c}^{-}$; the superconducting phases are denoted by $A$ and $B ; c / T$ vs. $T$ shows power-law behaviour below $T_{c}^{-}$instead of the usual exponential temperature dependence; data from Ref. [22].

Fig. 7. The superconducting phase diagram of $\mathrm{UPt}_{3}$ for field directions parallel and perpendicular to the hexagonal axis; three different superconducting phases are distinguished, denoted by $A, B$, and $C$; data from Ref. [26]. 
-pressure experiments revealed that the double transition in the specific heat disappears at around 4 kbar [27].

However, not only the double transition is sensitive to pressure. In neutron-diffraction experiments a clear signal of antiferromagnetism with moments of about $0.02 \mu_{\mathrm{B}}$ per formula unit is observed below $6 \mathrm{~K}$ [28]. Exactly this weak antiferromagnetic moment is suppressed by pressure and disappears as well around $4 \mathrm{kbar}$ [29]. This provoked the suggestion that the double transition is related to a symmetry-breaking field of magnetic origin. In subsequent years, different models within a Ginzburg-Landau description have been proposed for the occurrence of the multiple superconducting phase diagram of $\mathrm{UPt}_{3}[30-33]$. In the presence of weak antiferromagnetism with moments of the order of $0.02 \mu_{\mathrm{B}} / \mathrm{U}$-atom that point along the basal-plane $a^{*}$-axis, it is possible to imagine a symmetry-breaking field that causes a double superconducting transition in zero field and a multiple superconducting phase diagram with fields applied within the hexagonal plane. To argue that the same multiple phase diagram is also observable for applied fields along the hexagonal axis is, however, not straightforward. Nevertheless, this is what is found experimentally, although the values for the characteristic fields such as the tetracritical field and the upper critical field are rather different for field directions perpendicular and parallel to the hexagonal axis with a larger value for the tetracritical field along the hexagonal axis and a larger value for the upper critical field at zero temperature within the hexagonal plane. The different superconducting phases are supposed to exhibit different gap structures and different flux-line profiles. In addition, substitution studies showed that superconductivity and antiferromagnetism are closely related phenomena in the $\mathrm{U}(\mathrm{Pt}, \mathrm{Pd})_{3}$ system [34]. Alltogether, the compound $\mathrm{UPt}_{3}$ provides us with several interesting features: a unique multiple superconducting phase diagram, the coexistence of superconductivity and weak antiferromagnetism and, finally, a competition between superconductivity and long-range antiferromagnetic order. This competition is evident from the development of superconductivity and long-range antiferromagnetic order in $\mathrm{U}(\mathrm{Pt}, \mathrm{Pd})_{3}$ compounds for $\mathrm{Pd}$ concentrations below 10 at.\%. In the remaining part of this review these three intriguing features of $\mathrm{UPt}_{3}$ will be further discussed.

\section{The multiple superconducting phase diagram of $\mathrm{UPt}_{3}$}

The superconducting phase diagram of $\mathrm{UPt}_{3}$ in the $B-T$ plane has been studied in detail on pure as well as on substituted compounds. The main features of this phase diagram are now well established: a multicomponent phase diagram with three different superconducting phases, denoted by $A, B$, and $C$ that meet with the normal state in a tetracritical point. In fact, for all three principal directions of the magnetic field ( $\boldsymbol{B}\|\boldsymbol{a}, \boldsymbol{B}\| \boldsymbol{b}$ and $\boldsymbol{B} \| \boldsymbol{c}$ ) three different superconducting phases were found, with no significant anisotropy in the hexagonal plane. It should be noticed that the phases labelled $C$ for $\boldsymbol{B} \perp \boldsymbol{c}$ and $\boldsymbol{B} \| \boldsymbol{c}$ are not necessarily identical. The thermodynamic stability of the phase diagrams has been established and 
the four phase lines are likely to be of second order, although a weakly first-order $B C$ transition cannot be excluded. Van Dijk et al. [26] report the following values for $B$ and $T$ at the tetracritical point: $T_{\mathrm{tcr}}=0.39 \mathrm{~K}, B_{\mathrm{tcr}}=0.44 \mathrm{~T}$ for fields applied perpendicular to the hexagonal axis, $T_{\mathrm{tcr}}=0.35 \mathrm{~K}, B_{\mathrm{tcr}}=0.95 \mathrm{~T}$ for fields applied parallel to the hexagonal axis. The behaviour of the different phases under uniaxial pressure along the hexagonal axis has been studied in a further analysis of the thermal expansion and magnetostriction measurements.

Thermodynamic considerations result in the identification of the high-field, low-temperature phase $C$ as the more stable phase at applying uniaxial pressures along the hexagonal axis, see Figs. 8 and 9 . Sound-velocity measurements [35] as a function of temperature, magnetic field $(\boldsymbol{B} \| \boldsymbol{c})$ and uni-axial pressure $(p \| c)$ confirm the picture sketched in Fig. 9. Also in the $p_{\mathrm{c}}-T$ plane, a tetracritical point is
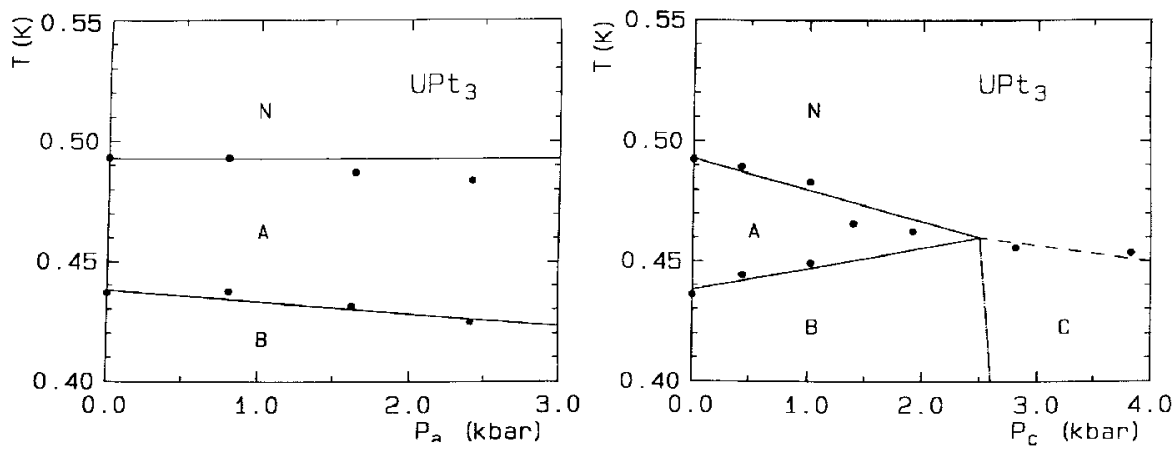

Fig. 8. The temperature-pressure superconducting phase diagram of $\mathrm{UPt}_{3}$ for uniaxial pressures along the $a$-axis (left part) and $c$-axis (right part); data from Ref. [26].

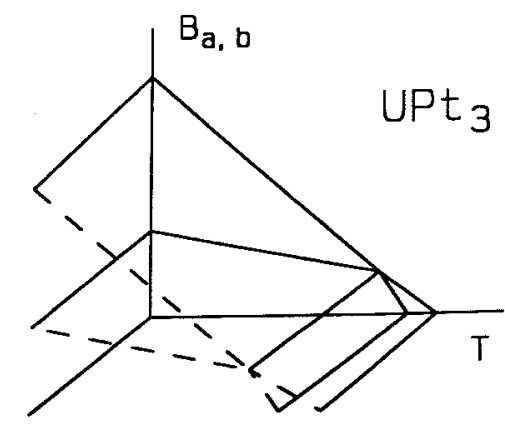

$p_{a}$

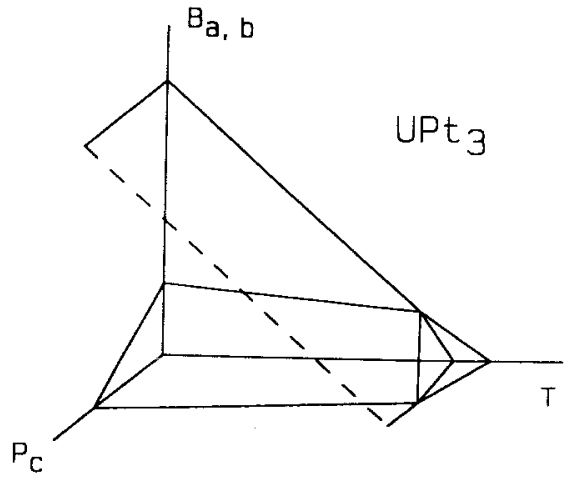

Fig. 9. The magnetic field-temperature-pressure superconducting phase diagram of $\mathrm{UPt}_{3}$ for uniaxial pressures along the $a$-axis (left part) and $c$-axis (right part) with magnetic fields in the hexagonal plane; data from Ref. [26]. 
found at $T_{\mathrm{tcr}}=0.46 \mathrm{~K}$ and $p_{\mathrm{tcr}}=2.5 \mathrm{kbar}$, whereas for hydrostatic pressures these values change into $0.43 \mathrm{~K}$ and $4.4 \mathrm{kbar}$, respectively. As was indicated above, this multicomponent phase diagram is often discussed in terms of a symmetry-breaking field that causes the splitting in the superconducting transition. A straightforward candidate for this symmetry-breaking field is the weak antiferromagnetism that has been observed in neutron experiments below $6 \mathrm{~K}$. Other candidates for this symmetry-breaking field, like a spontaneous lattice distortion or stacking faults in the atomic order, have been considered as well [36].

Additional studies have been reported for non-stoichiometric $\mathrm{UPt}_{3 \pm x} \mathrm{com}$ pounds with $x$-values of 0.06 in which no clear changes in the splitting at the superconducting transition are observed $[37,38]$. X-ray and microprobe measurements indicate the presence of small amounts of second phases in the non-stoichiometric compounds which is for the $x=0.06$ sample the neighbouring compound $\mathrm{UPt}_{5}$ and for the $x=-0.06$ sample the compound $\mathrm{UPt}_{2}$. Both neighbouring compounds do not show neither superconductivity nor magnetic order. The actual deviation from stoichiometry of the $x= \pm 0.06$ samples is considerably smaller than the nominal composition and is estimated to be of the order of $x= \pm 0.02$. There is little effect of the deviations of stoichiometry on the start of the superconducting transition and on the temperature splitting of the superconducting transition. The splitting is most sharply visible in the U-rich compound which might be related to a reduced oxygen content by the formation of uranium oxide as an impurity phase that does not affect the superconducting properties. From these results it is clear that impurity phases and deviations from stoichiometry play no decisive role in the transition to the superconducting state.

On the other hand, substitution studies result in all cases in a fast depression of superconductivity with, in case of $\mathrm{Pd}$ substitutions, an appreciable increase in the distance in temperature between the two superconducting transitions [39], see Fig. 10. Yttrium or thorium substitutions for uranium do not cause such an increase in this splitting. Other substitutions of magnetic ( $\mathrm{Gd}, \mathrm{Fe}, \mathrm{Co}, \mathrm{Ni}$ ) or non-magnetic ( $\mathrm{Au}, \mathrm{Ru}, \mathrm{Rh}, \mathrm{Ir}$ ) elements have not been studied in such detail but result in all cases in a depression of superconductivity with no special role of the magnetic substitutes [40], see Fig. 11. In these substitution studies it has been demonstrated that both magnetic and non-magnetic impurities suppress superconductivity at the same rate with respect to $\rho_{0}$, the residual resistivity. It is concluded from these experiments that magnetic pair breaking for these substitutions is not playing a significant role in the suppression of $T_{\mathrm{C}}$. This lack of spin-flip scattering contributions hints to parallel-spin electron pairing. On the other hand, in an analysis of substitution studies in $\mathrm{U}\left(\mathrm{Pt}_{1-x} \mathrm{Y}_{x}\right)_{3}, \mathrm{U}\left(\mathrm{Pt}_{1-x} \mathrm{Th}_{x}\right)_{3}$ and $\mathrm{U}\left(\mathrm{Pt}_{1-x} \mathrm{Pd}_{x}\right)_{3}$ Vorenkamp et al. [41] arrived at the conclusion that pair-breaking effects are dominant at depressing superconductivity for both yttrium and palladium substitutions. 


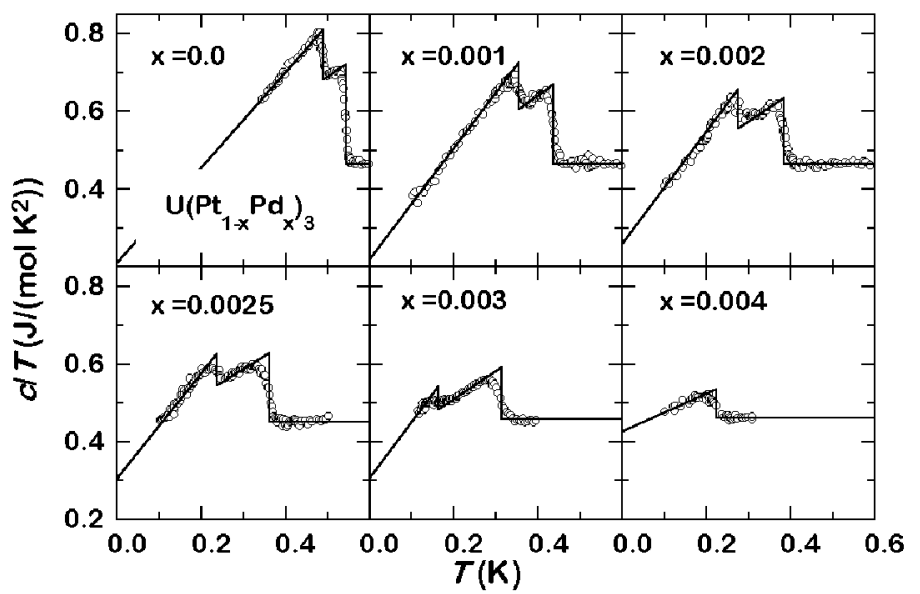

Fig. 10. $c / T$ vs. $T$ plots for $\mathrm{U}\left(\mathrm{Pt}_{1-x} \mathrm{Pd}_{x}\right)_{3}$ compounds with $x$-values indicated; the distance in temperature between the two superconducting transitions increases with increasing Pd content; data from Ref. [39].

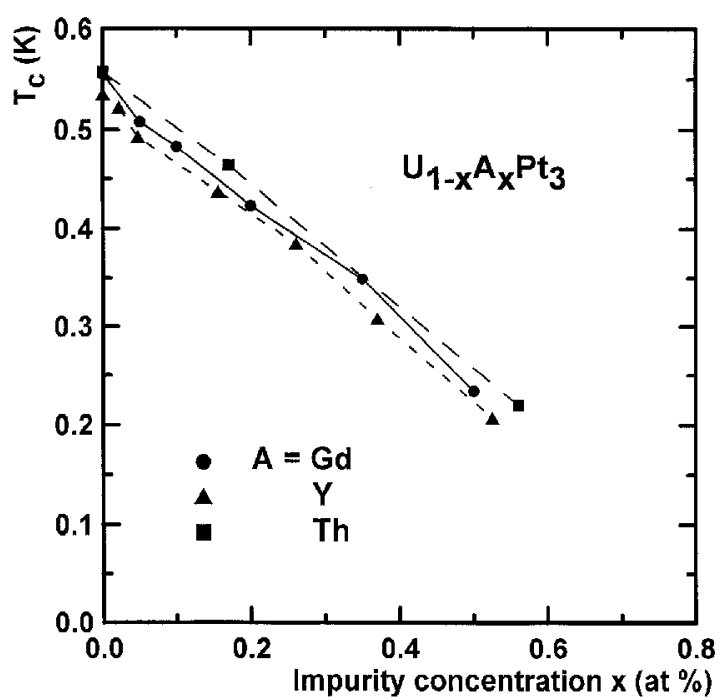

Fig. 11. The superconducting transition temperature, $T_{\mathrm{c}}$, of $\mathrm{UPt}_{3}$ as a function of magnetic (Gd) and non-magnetic (Y,Th) impurities; data from Ref. [40].

Another remarkable feature is worth mentioning here. In a more recent study of the suppression of superconductivity in single crystals of $\mathrm{UPt}_{3}$ by $\mathrm{Pd}$ substitution it was found that the residual resistivity for current directions along the hexagonal axis is systematically lower than for current directions in the hexago- 
nal plane [42]. Moreover, the resistive width of the superconducting transition is unaffected by $\mathrm{Pd}$ concentrations up to 0.002 for current along the $c$-axis, whereas for current along the $a$-axis the width increases by a factor more than two in the same concentration range. It seems to be natural to associate the anisotropy in $\rho_{0}$ with the magnetic fluctuation spectrum and the anisotropic broadening of the superconducting transition with the measured increase in the small antiferromagnetic moment which occurs for Pd substitution. Assuming a single- $q$ magnetic structure for the weak antiferromagnetic order with the local magnetic moments aligned parallel or antiparallel to the $a$-axis, one has to consider three equivalent magnetic domains in the $a-b$ plane for this structure. In this way it is very much possible that the broadening for current along the $a$-axis results from scattering at domain walls with an intensity that increases with increasing $\mathrm{Pd}$ content and, consequently, with the increase in strength of the weak antiferromagnetism. This does not occur for a current direction along the $c$-axis as all three magnetic domains are equivalent along this axis. In the next sections, the effects of $\mathrm{Pd}$ substitution on the magnetic behaviour will further be discussed. Here, we just notice that antiferromagnetism is enhanced by the Pd substitution and that these substitution studies, in which the splitting is found to be enhanced as well, confirm the nature of the symmetry-breaking field as being connected to the weak antiferromagnetism observed in neutron experiments.

The multiple superconducting phase diagram of $\mathrm{UPt}_{3}$ is not particularly robust against $\mathrm{Pd}$ substitution: the $B$ and $C$ phases are shifted towards lower temperatures much faster than the suppression of $T_{c}$ at $\mathrm{Pd}$ substitution. These particularities have been revealed in a detailed study of a single-crystalline $\mathbf{U}\left(\mathrm{Pt}_{0.998} \mathrm{Pd}_{0.002}\right)_{3}$ sample. Substitution of $\mathrm{Pt}$ by isoelectronic Pd leads in any case to a reduction of the elementary cell volume and to slight anisotropic changes in the lattice parameters. According to Van Sprang, the a-parameter is almost constant at Pd substitution up to 10 at.\% $\mathrm{Pd}$, whereas the c-parameter decreases at a rate of $(3.4 \pm 2.4) \times 10^{-4} \AA$ per at. $\% \mathrm{Pd}$ or $\Delta c / c=-(0.7 \pm 0.5) \times 10^{-4}$ per at. $\% \mathrm{Pd}$ [43]. Using the compressibility data deduced from sound-velocity measurements [44] we arrive at $\kappa_{c}=-(1 / c)(\mathrm{d} c / \mathrm{d} p)=0.151 \mathrm{Mbar}^{-1}$ and conclude that the effect of 1 at.\% $\mathrm{Pd}$ substitution is equivalent with a uniaxial pressure along the $c$-axis of about 2 kbar. In that sense, the results of both types of uniaxial pressure effects on the double superconducting transition (direct pressure and chemical pressure) point into different directions. The effect of $\mathrm{Pd}$ substitution is, of course, not just reducing the lattice parameter along the hexagonal axis. If that were the case, we had to expect that the increase in the width of the superconducting transition upon $\mathrm{Pd}$ substitution is accompanied by an increase in the superconducting transition temperature as well, according to the phase diagram in the $p_{\mathrm{c}}-T$ plane, depicted in Fig. 8. Pair-weakening or pair-breaking effects certainly are relevant at $\mathrm{Pd}$ substitution and will cause a reduction of $T_{\mathrm{c}}$. Comparing the relative volume changes at $\mathrm{Pd}$ substitution and at direct application of hydrostatic pressure, the effect of 
1 at.\% Pd substitution corresponds to a positive pressure of 0.15 kbar. However, comparing the relative changes in the $c / a$ ratio, the effect of 1 at.\% $\mathrm{Pd}$ substitution corresponds to a negative hydrostatic pressure of about $5 \mathrm{kbar}$. This suggests that the change of the $c / a$ ratio is more important for explaining the increase in the superconducting width at Pd substitution than changes in volume or $c$-parameter [45]. Similar conclusions have also been reached at analysing spin fluctuations and antiferromagnetism in $\mathrm{UPt}_{3}$ alloyed with $\mathrm{Th}, \mathrm{Au}$, and Ir impurities [46]. For instance, as the $c / a$ ratio decreases with Th and Au substitutions, spin fluctuations and antiferromagnetism are enhanced whereas with Ir substitution the $c / a$ ratio increases and the opposite behaviour is found for the magnetic properties. The effects on the metamagnetic transition induced by pressure (Fig. 3) or by Pd substitution (Fig. 1) and mentioned in the introduction support this suggestion.

Now the phase lines between the three different superconducting phases have well been established, the question arises which are the specific features that characterize the different superconducting phases. One of these features is the symmetry of the superconducting gap. Specific heat studies below the superconducting transition temperature show a power law temperature dependence which is indicative for point or line singularities in the superconducting gap function. In subsequent muon spin resonance ( $\mu \mathrm{SR})$ experiments these features have further been investigated. Broholm et al. [47] measured the anisotropy and temperature dependence of the magnetic field penetration by muon spin relaxation. The observed power-law temperature dependence of the penetration depth could be accounted for by a superconducting gap function with line nodes in the basal plane and axial point nodes. The present understanding is that the gap function shows different symmetries in the three phases which should have an impact on the symmetry of the flux line lattice in the different superconducting phases. Small angle neutron scattering from the flux line lattice of $\mathrm{UPt}_{3}$ in the different superconducting phases clearly reveals a transformation in the flux line lattice going from one superconducting phase to another indeed [48].

\section{Coexistence of superconductivity and weak antiferromagnetism in $\mathbf{U}\left(\mathbf{P t}_{1-x} \mathbf{P} \mathbf{d}_{x}\right)_{3}, x \leq 0.005$}

It has been recognized, for more than a decade now, that the heavy-fermion compound $\mathbf{U P t}_{3}$ is close to an antiferromagnetic instability. Evidence for the proximity to a magnetic instability is provided by pronounced spin-fluctuation phenomena at low temperatures and incipient order which becomes visible at chemical substitutions.

Inelastic neutron-scattering experiments $[28,49,50]$ have put the evidence for antiferromagnetic spin fluctuations on firm footing. The fluctuation spectrum is quite complex as different energy scales turn out to be present. Spin-polarized neutron-scattering data on polycrystalline material yield a quasi-elastic contribution centred at $\approx 10 \mathrm{meV}$, which is related to fluctuating local $f$ moments. 
The size of the fluctuating moment is of the order of $2 \mu_{\mathrm{B}} / \mathrm{U}$-atom, which is not far from the value of the effective moment for the high-temperature Curie-Weiss constant $\left(\mu_{\mathrm{eff}}=2.6 \pm 0.2 \mu_{\mathrm{B}} / \mathrm{U}\right.$-atom). Subsequent polarized and unpolarized neutron-scattering measurements on single-crystalline samples reveal a response centred at $5 \mathrm{meV}$, which is consistent with antiferromagnetic short-range order between nearest neighbour uranium atoms located in adjacent planes parallel to the hexagonal plane. The antiferromagnetic correlations disappear above the temperature where the maximum in the basal-plane susceptibility is found $(18 \mathrm{~K})$. At yet a lower energy $(0.2 \mathrm{meV})$ a second type of antiferromagnetic in-plane correlations was found at $\boldsymbol{Q}=(1 / 2,0,1)$, the wave vector where also weak magnetic Bragg reflections were detected. This then provided evidence that in $\mathbf{U P t}_{3}$ small-moment antiferromagnetic order develops below an ordering temperature of about $6 \mathrm{~K}$. The size of the ordered moment is unusually small, of the order of $0.02 \pm 0.01 \mu_{\mathrm{B}} / \mathrm{U}$-atom. It is directed along the $a^{*}$ axis in the hexagonal plane. The magnetic unit cell consists of a doubling of the nuclear unit cell along the $a^{*}$ axis. More recently, ferromagnetic correlations were observed near $\boldsymbol{Q}=0$, that persist till about $150 \mathrm{~K}$.

Superconductivity in $\mathrm{UPt}_{3}$ is suppressed by substitution of $\mathrm{Pt}$ by $\mathrm{Pd}$. At the same time the weak antiferromagnetism is enhanced as we already suggested in the previous section at discussing the increased splitting in the double superconducting transition for the $\mathrm{U}\left(\mathrm{Pt}_{0.998} \mathrm{Pd}_{0.002}\right)_{3}$ compound. The main question, of course, is whether the weak antiferromagnetism is indeed increased with $\mathrm{Pd}$ substitutions. In early experiments on samples with $x>0.02$, long-range antiferromagnetic order was found but this long-range order was certainly different in nature from the weak antiferromagnetism observed in pure $\mathbf{U P t}_{3}$. In order to shed further light on this question, a series of neutron-diffraction and muon spin relaxation studies has been started on monocrystalline $\mathrm{U}\left(\mathrm{Pt}_{1-x} \mathrm{Pd}_{x}\right)_{3}$ samples with $x$-values of $0.000,0.001,0.002$, and 0.005 . In Fig. 12 the temperature variation of the magnetic moment squared, $m^{2}(T)$, as derived from the maximum intensity of the antiferromagnetic Bragg peak at $\boldsymbol{Q}=(1 / 2,1,0)$, is shown for all four samples (after subtraction of the background) in terms of the square of the magnetic moment per uranium atom [51]. The behaviour of $m^{2}(T)$ for the various samples is quite unusual as far as its temperature dependence is concerned. The weak antiferromagnetism gradually develops below about $6 \mathrm{~K}$ which temperature does not seem to be dependent on the palladium concentration. The values of $m^{2}$ start to rise slowly below $6 \mathrm{~K}$ and exhibit a quasi-linear temperature dependence down to the temperature where superconductivity develops. In the superconducting state, the magnetic intensities saturate. The zero-temperature values for $\mathrm{m}^{2}$ increase with increasing $\mathrm{Pd}$ substitution and confirm the picture that was developed in the previous section. The absolute values of the ordered moments have been calculated using integrated intensities. Values for $m\left(T_{c}^{+}\right)$of $18,24,36$, and $48 \times 10^{-3} \mu_{\mathrm{B}} / \mathrm{U}$-atom are derived for $x=0.000,0.001,0.002$, and 0.005 , respec- 
tively. The experimental data of Fig. 12 do not point to a magnetic phase transition at around $6 \mathrm{~K}$. The picture that arises is a gradual development of antiferromagnetic correlations upon lowering temperatures below $6 \mathrm{~K}$. The effect on annealing was investigated for the $x=0.001$ and 0.002 samples. It was found that the size of the ordered moment does not change (within the experimental accuracy) by the annealing procedures. In order to investigate the effect of annealing on the magnetic correlation length, $\xi_{\mathrm{m}}$, the magnetic Bragg peak at $\boldsymbol{Q}=(1 / 2,1,0)$ has been scanned as a function of $Q$ at several selected temperatures for $x=0.001$ and 0.002 before and after annealing the samples. By fitting the profiles, values for the correlation length, $\xi_{\mathrm{m}}$, of about $600 \AA$ could be derived. Since the size of the ordered moment and the values of the correlation lengths are within the experimental error the same for annealed and unannealed samples, one can conclude that the weak antiferromagnetism is not particularly sensitive to strain effects in the samples.

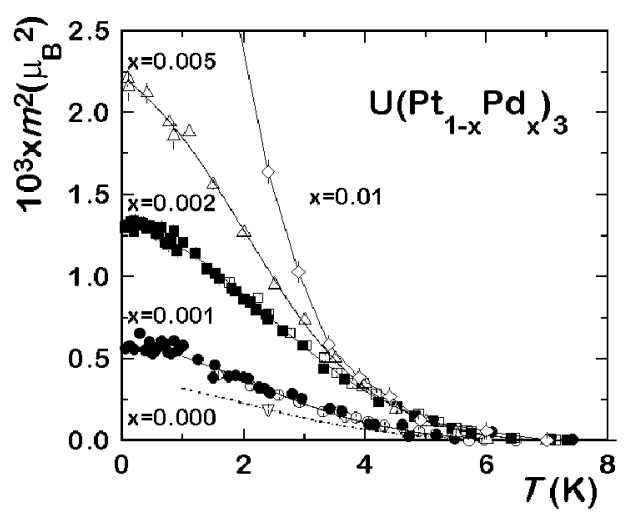

Fig. 12. Temperature variation of $m^{2}$ derived from the intensity of the magnetic Bragg peak at $\boldsymbol{Q}=(1 / 2,1,0)$ for annealed (open symbols) and unannealed (closed symbols) $\mathrm{U}\left(\mathrm{Pt}_{1-x} \mathrm{Pd}_{x}\right)_{3}$ samples with $x$-values up to 0.01 ; the solid lines are guides to the eye; data from Ref. [51].

At higher Pd concentration, long-range static magnetic order develops. The neutron diffraction results for single-crystalline samples with $\mathrm{Pd}$ concentrations of $0.01,0.02$, and 0.05 will be reviewed in the next section.

Muon spin depolarization studies have been employed to further investigate the magnetic behaviour of these compounds. In early $\mu \mathrm{SR}$ experiments, a weak static moment of the order of $10^{-3}-10^{-2} \mu_{\mathrm{B}} / \mathrm{U}$-atom was reported in $\mathrm{UPt}_{3}$ [52]. More recently, $\mu \mathrm{SR}$ experiments have been carried out on the $x=0.000$ and 0.002 samples in order to examine the evolution of magnetism on the microscopic level in the superconducting $\mathrm{U}(\mathrm{Pt}, \mathrm{Pd})_{3}$ compounds $[53,54]$. Zero-field data have been taken on pure $\mathrm{UPt}_{3}$ in the temperature interval $2.7-7.0 \mathrm{~K}$ and for the $x=0.002$ compound in the $T$-interval $0.9-8.0 \mathrm{~K}$. Surprisingly, the more recent data differ 
from the earlier results and did not confirm any weak static type of magnetic order in both samples. One conclusion from these experiments could be that the stopping site of the muon in $\mathrm{UPt}_{3}$ is non-magnetic by cancellation of different contributions to the internal magnetic field. However, this is highly unlikely as all compounds with $x$-values up to 5 at.\% Pd have identical magnetic and crystallographic struc-

tures. We anticipate on the discussion in the next section by noting that for higher $\mathrm{Pd}$ concentrations where long-range antiferromagnetic order is observed, the $\mu \mathrm{SR}$ experiments clearly reveal magnetic order below $T_{\mathrm{N}}$ and that on the basis of the moments derived from neutron diffraction, a $\mu \mathrm{SR}$ signal in pure $\mathrm{UPt}_{3}$ had to be expected. An alternative interpretation for the absence of magnetic signals in the $\mu \mathrm{SR}$ experiments on $\mathrm{UPt}_{3}$ assumes that the weak antiferromagnetic moments in $\mathrm{UPt}_{3}$ observed in neutron diffraction are not static but rather fluctuate at a rate larger than $10 \mathrm{MHz}$, a frequency too high for the $\mu \mathrm{SR}$ experiments but low enough to observe these moments in neutron scattering experiments [51]. This suggestion could also solve the longstanding problem that the small moment of $\mathrm{UPt}_{3}$ has not been observed in NMR measurements [55], while its signal should well fall in the detection limit as was concluded from experiments at higher $\mathrm{Pd}$ concentrations which successfully probed the long-range antiferromagnetic order.

In the Ginzburg-Landau description of the multiple superconducting phase diagram we discussed the possibility of a symmetry-breaking field of magnetic origin. Neutron scattering experiments were mentioned that support this scenario. However, the existing neutron-scattering data do not distinguish between a single- $\boldsymbol{q}$ structure with three magnetic domains and a triple- $\boldsymbol{q}$ structure. The question whether the magnetic order corresponds to a single- $\boldsymbol{q}$ or a triple- $\boldsymbol{q}$ structure is crucial for the understanding of the unconvential superconductivity. A single- $\boldsymbol{q}$ structure breaks the hexagonal symmetry while a triple- $\boldsymbol{q}$ structure does not. The two structures can be distinguished by applying a magnetic field. Experiments performed up to $12 \mathrm{~T}$ did not show any redistribution of magnetic domains indicating that a triple- $\boldsymbol{q}$ structure cannot be excluded. However, it is possible that a field of $12 \mathrm{~T}$ is not sufficiently strong to change the domain population of moments as weak as $0.02 \pm 0.01 \mu_{\mathrm{B}} / \mathrm{U}$-atom. This possibility is supported by the fact that the weak antiferromagnetism is very stable in applied magnetic fields. The temperature below which the weak antiferromagnetism is observed is only slightly depressed $(\approx 0.5 \mathrm{~K})$ in a magnetic field of $10 \mathrm{~T}$. Neutron-diffraction experiments on $\mathbf{U}\left(\mathrm{Pt}_{0.95} \mathrm{Pd}_{0.05}\right)_{3}$ in an applied field of $5 \mathrm{~T}$ revealed the formation of a single-domain sample in $5 \mathrm{~T}[56]$.

\section{Competition between superconductivity and long-range antiferromagnetic order in the $\mathrm{U}(\mathbf{P t}, \mathbf{P d})_{3}$ system}

Specific heat studies on $\mathrm{U}\left(\mathrm{Pt}_{1-x} \mathrm{Pd}_{x}\right)_{3}$ compounds for $x$-values between 0.01 and 0.10 point to the presence of long-range antiferromagnetic order with a maximal value for $T_{\mathrm{N}}$ of about $6 \mathrm{~K}$ for $x=0.05$ with borders of the antiferromagnetic 
order at $x$-values of about 0.01 and 0.10 . In neutron diffraction experiments the type of order has been established for $x=0.05$ [57]. In addition, pronounced phase-transition anomalies were found at the Néel temperature of $5.8 \mathrm{~K}$ in the thermal and transport properties of the $x=0.05$ compound. Notably, the $\lambda$-like anomaly in $c(T)$ and the Cr-type anomaly in $\rho(T)$ give evidence for an antiferromagnetic phase transition of the spin-density-wave type. By plotting the Néel temperatures, deduced from the $c(T)$ and $\rho(T)$ data as function of the $\mathrm{Pd}$ concentration, the border of the antiferromagnetic phase could be evidenced [58]. The proximity of long-range antiferromagnetic order is a general feature of heavy-fermion systems and the compound $\mathbf{U P t}_{3}$ is not an exception. This magnetic instability is usually explained in terms of a competition between the on-site Kondo effect and the intersite Ruderman-Kittel-Kasuya-Yoshida (RKKY) interaction [59]. However, in the case of the $\mathbf{U}\left(\mathrm{Pt}_{1-x} \mathrm{Pd}_{x}\right)_{3}$ system, a clear-cut identification of $T_{\mathrm{K}}$ and $T_{\text {RKKY }}$ is not at hand [60]. In the recent neutron-diffraction experiments discussed before, the disappearance of the antiferromagnetic order for $x$-values below 0.02 has been investigated in detail [51]. The neutron-diffraction experiments on pure $\mathrm{UPt}_{3}$ and on $\mathrm{U}\left(\mathrm{Pt}_{0.95} \mathrm{Pd}_{0.05}\right)_{3}$ show that the magnetic structure is in both cases the same. The magnetic unit cell corresponds to a doubling of the nuclear unit cell along the $a^{*}$-axis with the moments pointing along the $a^{*}$-axis, see Fig. 13 . In Fig. 14, the temperature variation of $m^{2}$ is shown for the $x$-values of $0.01,0.02$, and 0.05 . The temperature variation of $m^{2}$ for $x=0.02$ and 0.05 is rather conventional compared to the quasi-linear temperature variation observed for the lower $x$-values in the previous section. The temperature variation of $m^{2}$ in the case of samples shown in Fig. 14 follows a $\left[1-\left(T / T_{\mathrm{N}}\right)^{\alpha}\right]^{2 \beta}$ dependence, where $\alpha$ reflects spin-wave excitations and where $\beta$ points to a $3 \mathrm{D}$ Heisenberg exchange interaction. Values for the extrapolated zero-temperature moments are 0.35 and $0.63 \mu_{\mathrm{B}} / \mathrm{U}$-atom for $x=0.02$ and 0.05 , respectively.

The temperature dependence of the magnetic Bragg intensity of the sample with $x=0.01$ is quite intriguing: $m^{2}(T)$ starts to rise slowly below $6 \mathrm{~K}$ and rises sharply below $2 \mathrm{~K}$, saturating below $0.5 \mathrm{~K}$. These results prove that the weak antiferromagnetism that is associated with antiferromagnetic fluctuations still exists in this compound and is even enhanced compared to the lower $x$-values compounds. The sharp rise below $2 \mathrm{~K}$, on the other hand, indicates the onset of long-range antiferromagnetic order with a saturated moment below $0.5 \mathrm{~K}$ of $0.11 \mu_{\mathrm{B}} / \mathrm{U}$-atom. The estimated value for the Néel temperature, $T_{\mathrm{N}}$, amounts to $1.8 \mathrm{~K}$ for the long-range antiferromagnetic order. This interpretation of a crossover from antiferromagnetic fluctuations that develop below $6 \mathrm{~K}$ to a long-range antiferromagnetically ordered state below $1.8 \mathrm{~K}$ is consistent with $\mu \mathrm{SR}$ experiments on $\mathrm{U}\left(\mathrm{Pt}_{0.99} \mathrm{Pd}_{0.01}\right)_{3}$ which show below $1.8 \mathrm{~K}$ a spontaneous $\mu^{+}$precession. This demonstrates that the weak antiferromagnetism and the long-range antiferromagnetic order are not directly connected. In macroscopic measurements, this transition at $1.8 \mathrm{~K}$ does not show up, in contrast to the thermal and transport data for the $x=0.02$ and 0.05 


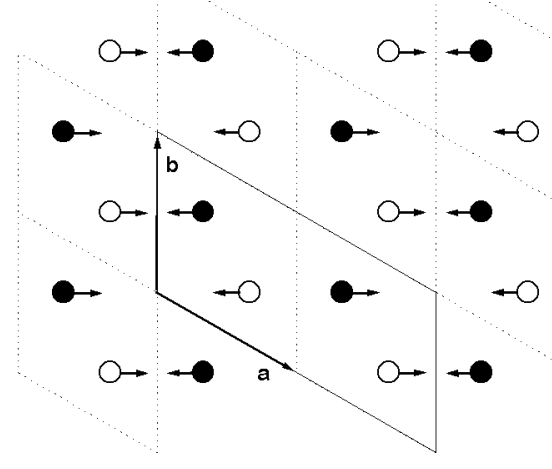

Fig. 13

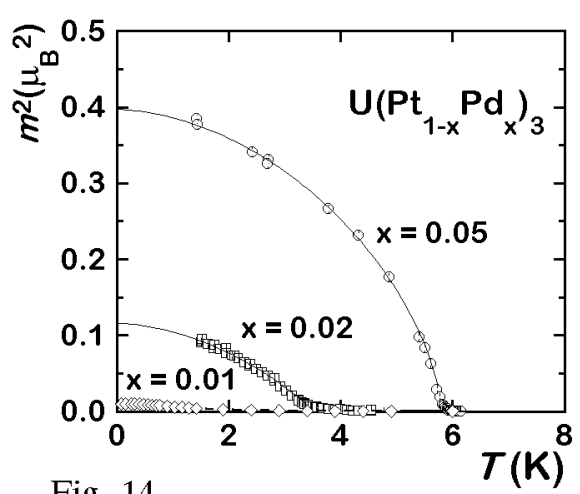

Fig. 14

Fig. 13. Magnetic structure of $\mathrm{UPt}_{3}$. The open and closed circles indicate $U$ atoms in adjacent hexagonal planes separated by a lattice spacing $c / 2$. The arrows indicate the magnetic moments, which are directed along the $a^{*}$-axis. The dotted and solid lines delineate the nuclear and magnetic unit cells, respectively [51].

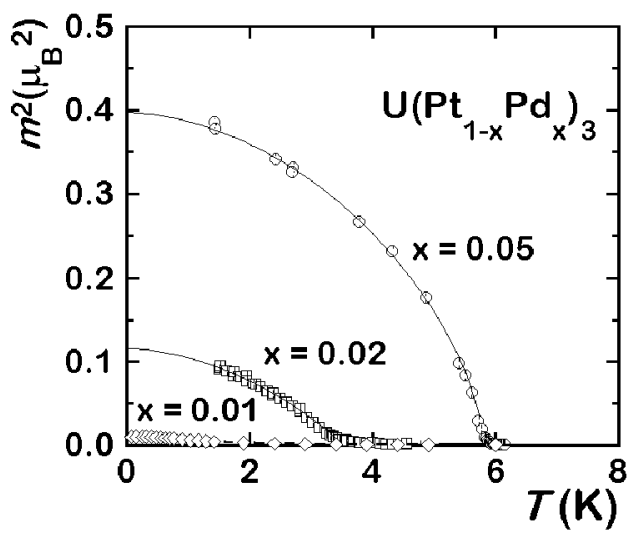

Fig. 14. Temperature variation of $m^{2}$ for annealed $\mathrm{U}\left(\mathrm{Pt}_{1-x} \mathrm{Pd}_{x}\right)_{3}$ samples as derived from the intensity of the magnetic Bragg peak at $\boldsymbol{Q}=(1 / 2,1,0)$ for $x=0.02$ and 0.05 and at $\boldsymbol{Q}=(1 / 2,0,1)$ for $x=0.01$; the solid lines represent fits to the expression $\left[1-\left(T / T_{\mathrm{N}}\right)^{\alpha}\right]^{2 \beta}$, see text; data from Ref. [51].

compounds in which, as mentioned before, magnetic phase transitions are clearly observed in specific heat measurements at $3.5 \mathrm{~K}$ and $5.8 \mathrm{~K}$, respectively.

Zero-field $\mu \mathrm{SR}$ experiments have been carried out on $\mathrm{U}\left(\mathrm{Pt}_{1-x} \mathrm{Pd}_{x}\right)_{3}$ samples with $x$-values of 0.01 [61], 0.02 [61] and 0.05 [53] which reveal spontaneous precession frequencies below the Néel temperatures of $1.8 \mathrm{~K}, 3.5 \mathrm{~K}$, and $6.3 \mathrm{~K}$, respectively, see also Ref. [54]. Below the ordering temperature of $6.3 \mathrm{~K}$, a value for $T_{\mathrm{N}}$ that slightly deviates from the neutron and specific heat results, the relaxation spectra have been analysed with two precession frequencies with extrapolated val- 
ues to zero temperature of 8.0 and $2.0 \mathrm{MHz}$. These two frequency components could indicate two magnetically inequivalent stopping sites. From the observed intensities it is then concluded that muons stop at both sites with the same probability. In further studies of the muon localization site, the angular and temperature $(10-250 \mathrm{~K})$ variation of the Knight shift of single-crystalline $\mathrm{U}\left(\mathrm{Pt}_{0.95} \mathrm{Pd}_{0.05}\right)_{3}$ has been measured in transverse-field $(B=0.6 \mathrm{~T}) \mu \mathrm{SR}$ experiments. By analysing the temperature variation of the Knight shift with a modified Curie-Weiss expression, the muon localization site in this hexagonal material is determined as a single site at $(0,0,0)$, in contrast to the above suggestion of two different stopping sites. Anyhow, there is still some uncertainty in the analysis of the $\mu$ SR spectra in terms of two different frequencies [62], see also Ref. [63].

In Fig. 15, the temperature dependence of the muon depolarization rate is plotted for $x$-values of $0.01,0.02$, and 0.05 [64]. The curves show some similarity with the $m^{2}$ curves of Fig. 14. Transverse field $\mu \mathrm{SR}$ measurements have been performed in an applied transverse field of 100 Gs on samples with $x$-values of 0.007 , 0.008 , and 0.009 at temperatures below $2 \mathrm{~K}$ [65]. The temperature variation of the exponential relaxation rate shows a temperature independent background and a quasilogarithmic increase below a temperature that is identified as the long-range antiferromagnetic ordering temperature, see Fig. 16. Values for $T_{\mathrm{N}}$ derived from the onset of the quasilogarithmic term equal $0.45 \mathrm{~K}, 0.78 \mathrm{~K}$, and $1.23 \mathrm{~K}$ for $x$-values of $0.007,0.008$, and 0.009 , respectively. In Fig. 17 the transition temperatures of the superconducting state and the long-range antiferromagnetic order are given [65].

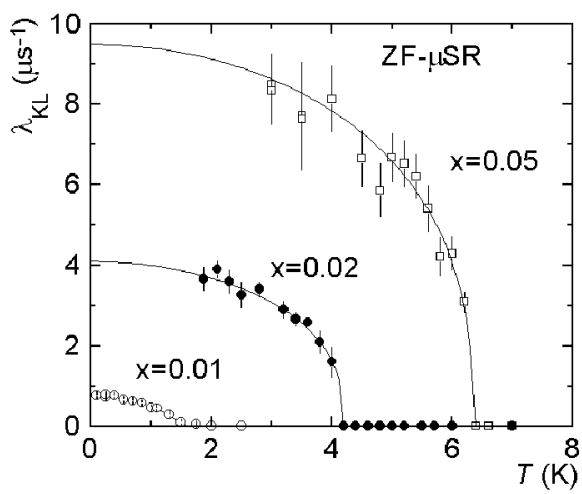

Fig. 15. Temperature dependence of the exponential and Lorentzian Kubo-Toyate relaxation rates, extracted from transverse field $\mu \mathrm{SR}$ spectra, for the long-range antiferromagnetically ordered $\mathrm{U}\left(\mathrm{Pt}_{1-x} \mathrm{Pd}_{x}\right)_{3}$ compounds with $x$-values of $0.01,0.02$, and 0.05 ; the solid line represents a fit to $f(T)=f(0)\left[1-\left(T / T_{\mathrm{N}}\right)^{\alpha}\right]^{\beta} ; \lambda_{\mathrm{KL}}(T)$ roughly scales with the ordered moment as deduced from the neutron-diffraction measurements; data from Ref. [64]. 


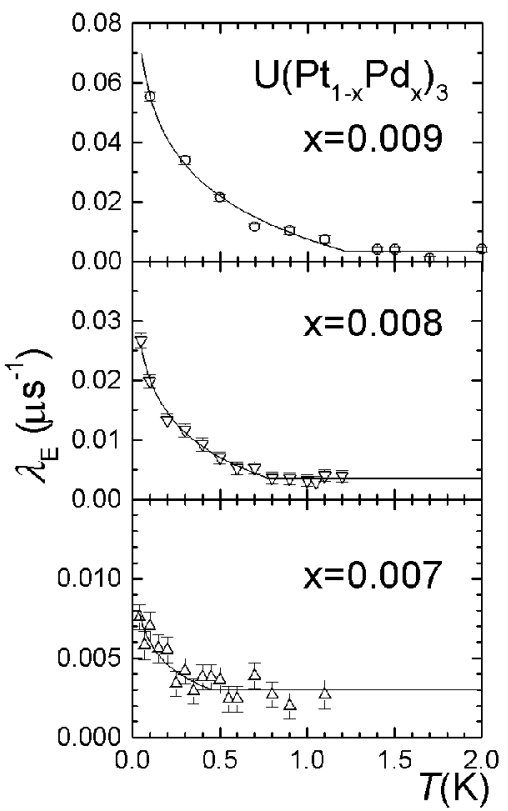

Fig. 16. Temperature dependence of the exponential relaxation rate, extracted from transverse field $(100 \mathrm{Gs}) \mu \mathrm{SR}$ spectra for $\mathrm{U}\left(\mathrm{Pt}_{1-x} \mathrm{Pd}_{x}\right)_{3}$ samples with $x=0.007,0.008$, and 0.009 . The solid lines show the quasilogarithmic increase below $T_{\mathrm{N}}$ and the temperature independent background above $T_{\mathrm{N}}$; data from Ref. [65].

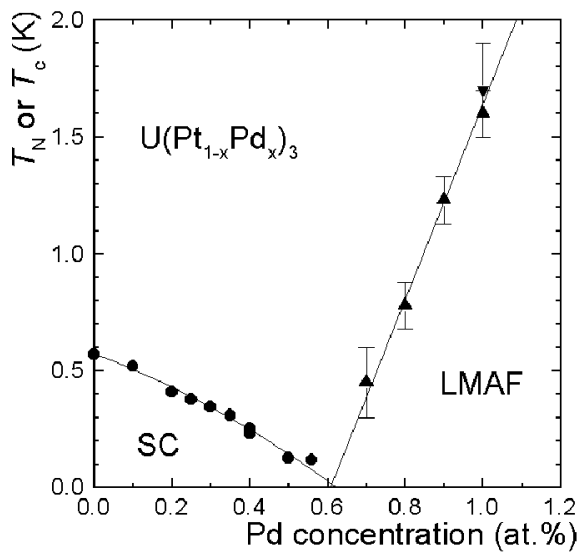

Fig. 17. Low-temperature phase diagram of the $\mathrm{U}\left(\mathrm{Pt}_{1-x} \mathrm{Pd}_{x}\right)_{3}$ system near the critical compositions for superconductivity, $x_{\mathrm{C}}^{\mathrm{SC}}$, and for long-range antiferromagnetic order, $x_{c}^{\mathrm{AF}}$; data from Ref. [65].

We can notice that superconductivity disappears where long-range antiferromagnetic order is starting, with an estimated value for the critical concentration of long-range antiferromagnetic order of about 0.6 at.\% $\mathrm{Pd}$. 


\section{Concluding remarks}

In the past fifteen years a wealth of experimental data have been provided for the electronic properties of the heavy-fermion superconductor $\mathrm{UPt}_{3}$. Salient features that emerge from these data are: the co-existence of superconductivity with weak antiferromagnetism in pure $\mathrm{UPt}_{3}$; the multiple superconducting phase diagram of $\mathrm{UPt}_{3}$ that, apparently, is connected to the weak antiferromagnetism; the competition between superconductivity and long-range antiferromagnetic order in $\mathrm{U}\left(\mathrm{Pt}_{1-x} \mathrm{Pd}_{x}\right)_{3}$ compounds resulting in a critical composition near $x=0.006$; the presence of both weak antiferromagnetism and long-range antiferromagnetic order at least in the $\mathrm{U}\left(\mathrm{Pt}_{1-x} \mathrm{Pd}_{x}\right)_{3}$ compound with $x=0.01$; the pseudo-metamagnetism in pure $\mathrm{UPt}_{3}$ at $20 \mathrm{~T}$ and the development from pseudo-metamagnetism towards metamagnetic behaviour in the long-range ordered $\mathrm{U}\left(\mathrm{Pt}_{1-x} \mathrm{Pd}_{x}\right)_{3}$ compounds at lower field values. The co-existence and competition between superconductivity and weak and strong antiferromagnetism, respectively, is summarized in Fig. 18 in which the results are shown for Pd concentrations up to 10 at.\%. At the higher concentration side of Fig. 18 we note that antiferromagnetic order is lost for $x=0.10$. Indeed, no long-range antiferromagnetic order has been observed for this compound. There is a lack, however, of information on the presence of weak antiferromagnetism for Pd concentrations above $x=0.01$. Additional neutron-diffraction and $\mu \mathrm{SR}$ experiments would be most welcome for that part of the phase diagram. On the other hand, one should keep in mind that the $\mathrm{MgCd}_{3}$-type of structure is lost for higher palladium concentrations as additional lines in the X-ray-diffraction patterns for $x \geq 0.15$ indicate.

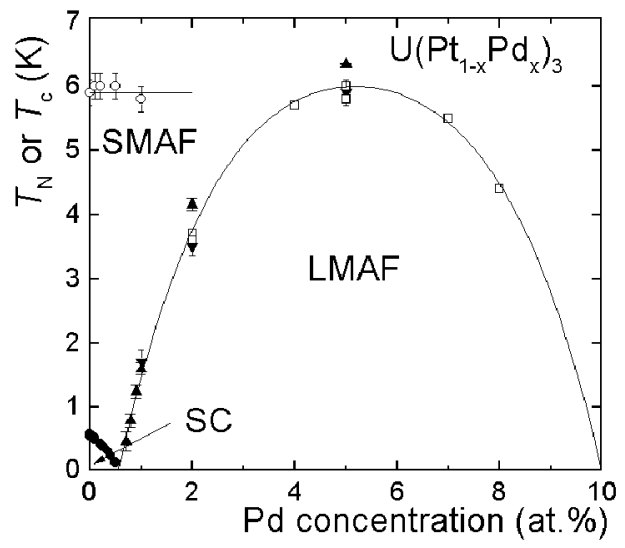

Fig. 18. Superconducting and antiferromagnetic ordering temperatures as function of concentration in the $\mathrm{U}\left(\mathrm{Pt}_{1-x} \mathrm{Pd}_{x}\right)_{3}$ system; $\mathrm{SC}$ denotes superconductivity, $\mathrm{SMAF}-$ small-moment antiferromagnetism, and LMAF — long-range antiferromagnetic order; data from Ref. [65]. 
At the left hand side of the phase diagram superconductivity and long-range antiferromagnetic order meet at the critical composition of about 0.6 at.\% Pd. Currently, much attention in heavy-fermion research is focussed on the occurrence of non-Fermi-liquid behaviour at this critical concentration. So far, signatures of non-Fermi-liquid properties have been observed in resistivity measurements on $\mathrm{U}\left(\mathrm{Pt}_{1-x} \mathrm{Pd}_{x}\right)_{3}$ compounds near the critical concentration [66]. The resistivity below $1 \mathrm{~K}$ for $0<x \leq 0.02$ shows a deviation from Fermi liquid behaviour described by $\rho(T)=\rho_{0}+A T^{\alpha}$ with $\alpha$ varying between 2 for $x=0$ and 1.6 for $x \approx x_{\mathrm{c}}$. This latter value for the power $\alpha$ indicates non-Fermi liquid behaviour and suggests that a quantum phase transition exists near $x_{\mathrm{c}}$. The nature of the critical point in the $\mathrm{U}\left(\mathrm{Pt}_{1-x} \mathrm{Pd}_{x}\right)_{3}$ system has further been explored in Ref. [65] where it is concluded that the existence of a magnetic quantum critical point in the phase diagram, which coincides with the critical point for superconductivity, provides evidence for ferromagnetic spin-fluctuation-mediated odd-parity superconductivity, which competes with long-range antiferromagnetic order. At applying pressure we move away from the coinciding quantum critical points as both superconductivity and antiferromagnetic order are depressed. Further studies near this critical composition are in progress [62].

\section{Acknowledgments}

The author is grateful to Dr. A. De Visser for his help at preparing the manuscript.

\section{References}

[1] P.H. Frings, J.J.M. Franse, F.R. de Boer, A. Menovsky, J. Magn. Magn. Mater. 31-34, 240 (1983).

[2] J.J.M. Franse, J. Magn. Magn. Mater. 31-34, 819 (1983).

[3] C.F. Kamma, P.H. Frings, J.C.P. Klaasse, J.J.M. Franse, Physica B 119, 72 (1983).

[4] P.H. Frings, J.J.M. Franse, J. Magn. Magn. Mater. 51, 141 (1985).

[5] P.H. Frings, J.J.M. Franse, Phys. Rev. B 31, 4355 (1985).

[6] J.J.M. Franse, M. van Sprang, A. de Visser, A.A. Menovsky, Physica B 163, 511 (1990).

[7] A. de Visser, L. Puech, W. Joss, A.A. Menovsky, J.J.M. Franse, Jpn. J. Appl. Phys. A 26, Supplement 26-3, 513 (1987).

[8] M. van Sprang, R.A. Boer, A.J. Riemersma, L.W. Roeland, A.A. Menovsky, J.J.M. Franse, J. Schoenes, J. Magn. Magn. Mater. 76-77, 229 (1988).

[9] K. Bakker, A. de Visser, A.A. Menovsky, J.J.M. Franse, Phys. Rev. B 46, 544 (1992)

[10] I. Kouroudis, D. Nikl, M. Yoshizawa, W. Assmus, B. Lüthi, J.J.M. Franse, A.A. Menovsky, U. Welp, G. Bruhls, J. Magn. Magn. Mater. 63-64, 389 (1987). 
[11] G.R. Stewart, Z. Fisk, J.L. Smith, J.J.M. Franse, A. Menovsky, B.L. Brandt, J. Magn. Magn. Mater. 76-77, 484 (1988).

[12] H.P. van der Meulen, Z. Tarnawski, J.J.M. Franse, J.A.A.J. Perenboom, D. Althof, H. van Kempen, Phys. Rev. B 41, 9352 (1990).

[13] J.J.M. Franse, A. de Visser, A. Menovsky, P.H. Frings, J. Magn. Magn. Mater. 52, 61 (1985).

[14] J.J.M. Franse, M. van Sprang, E. Louis, K. Kadowaki, A. de Visser, J. Magn. Magn. Mater. 76-77, 147 (1988).

[15] J.J.M. Franse, M. van Sprang, A. de Visser, P.E. Brommer, Physica B 154, 379 (1989).

[16] R. Verhoef, A. de Visser, A. Menovsky, A.J. Riemersma, J.J.M. Franse, Physica B 142, 11 (1986).

[17] A. Menovsky, J.J.M. Franse, A.C. Moleman, J. Cryst. Growth 67, 31 (1984).

[18] G.R. Stewart, Z. Fisk, J.O. Willis, J.L. Smith, Phys. Rev. Lett. 52, 679 (1984).

[19] F. Steglich, J. Aarts, C.D. Bredl, W. Lieke, D. Meschede, W. Franz, H. Schäfer, Phys. Rev. Lett. 43, 1892 (1979).

[20] H.R. Ott, H. Rudigier, Z. Fisk, J.L. Smith, Phys. Rev. Lett. 50, 1595 (1983).

[21] R.A. Fisher, S. Kim, B.F. Woodfield, N.E. Phillips, L. Taillefer, K. Hasselbach, J. Flouquet, A.L. Giorgi, J.L. Smith, Phys. Rev. Lett. 62, 1411 (1989).

[22] K. Hasselbach, L. Taillefer, J. Flouquet, Phys. Rev. Lett. 63, 93 (1989).

[23] L. Taillefer, R. Newbury, G.G. Lonzarich, Z. Fisk, J.L. Smith, J. Magn. Magn. Mater. 63-64, 372 (1987).

[24] U. Rauchschwalbe, U. Ahlheim, F. Steglich, D. Rainer, J.J.M. Franse, Z. Phys. B 60, 379 (1985).

[25] N.H. van Dijk, A. de Visser, J.J.M. Franse, S. Holtmeier, L. Taillefer, J. Flouquet, Physica B 186-188, 267 (1993); Phys. Rev. B 48, 1299 (1993).

[26] N.H. van Dijk, A. de Visser, J.J.M. Franse, L. Taillefer, J. Low Temp. Phys. 93, 101 (1993).

[27] T. Trappmann, H. von Löhneysen, L. Taillefer, Phys. Rev. B 43, 13714 (1991).

[28] G. Aeppli, E. Bucher, C. Broholm, J.K. Kjems, J. Baumann, J. Hufnagl, Phys. Rev. Lett. 60, 615 (1988).

[29] S.M. Hayden, L. Taillefer, C. Vettier, J. Flouquet, Phys. Rev. B 46, 8675 (1992).

[30] J.A. Sauls, Adv. Phys. 43, 113 (1994).

[31] R. Joynt, Supercond. Sci. Technol. 1, 210 (1988).

[32] K. Machida, M. Ozaki, T. Ohmi, J. Phys. Soc. Jpn. 58, 4116 (1989).

[33] D.W. Hess, T. Tokuyasu, J.A. Sauls, J. Phys., Condens. Matter 1, 8135 (1989).

[34] J.J.M. Franse, N.H. van Dijk, A. de Visser, U. Wyder, Physica B 223-224, 15 (1996).

[35] M. Boukhny, G.L. Bullock, B.S. Shivaram, D.G. Hinks, Phys. Rev. Lett. 73, 1707 (1994).

[36] M.C. Aronson, R. Clarke, B.G. Demczyk, B.R. Coles, J.L. Smith, A. de Visser, T. Vorenkamp, J.J.M. Franse, Physica B 186-188, 788 (1993). 
[37] K. Bakker, A. de Visser, J.J.M. Franse, J. Magn. Magn. Mater. 108, 65 (1992).

[38] J.J.M. Franse, K. Bakker, N.H. van Dijk, A.A. Menovsky, A de Visser, J. Low-Temp. Phys. 95, 177 (1994).

[39] R.J. Keizer, A. de Visser, M.J. Graf, A.A. Menovsky, J.J.M. Franse, Phys. Rev. B 60, 10527 (1999).

[40] H.G.M. Duijn, N.H. van Dijk, A. de Visser, J.J.M. Franse, Physica B 223-224, 44 (1996).

[41] T. Vorenkamp, M.C. Aronson, Z. Koziol, K. Bakker, J.J.M. Franse, J.L. Smith, Phys. Rev. B 48, 6373 (1993).

[42] M.J. Graf, R.J. Keizer, A. de Visser, A.A. Menovsky, J.J.M. Franse, Phys. Rev. B 60, 3056 (1999).

[43] M. van Sprang, Ph. D. thesis, University of Amsterdam, 1989.

[44] A. de Visser, J.J.M. Franse, Physica B 130, 177 (1985).

[45] K. Bakker, Ph. D. thesis, University of Amsterdam, 1993.

[46] B. Batlogg, D.J. Bishop, E. Bucher, B. Golding, A.P. Ramirez, Z. Fisk, J.L. Smith, H.R. Ott, J. Magn. Magn. Mater. 63-64, 441 (1987).

[47] C. Broholm, G. Aeppli, R.N. Kleinman, D.R. Harshman, D.J. Bishop, E. Bucher, D.L. Williams, E.J. Ansaldo, R.H. Heffner, Phys. Rev. Lett. 65, 2062 (1990).

[48] A. Huxley, P. Rodière, D. McK. Paul, N.H. van Dijk, R. Cubitt, J. Flouquet, Nature 406, 160 (2000).

[49] G. Aeppli, E. Bucher, G. Shirane, Phys. Rev. B 32, 7579 (1985).

[50] G. Aeppli, A. Goldman, G. Shirane, E. Bucher, M.-Ch. Lux-Steiner, Phys. Rev. Lett. 58, 808 (1987).

[51] R.J. Keizer, A. de Visser, A.A. Menovsky, J.J.M. Franse, B. Fåk, J.-M. Mignot, Phys. Rev. B 60, 6668 (1999).

[52] R. Heffner, D.W. Cooke, A.L. Giorgi, R.L. Hutson, M.E. Schillaci, H.D. Rempp, J.L. Smith, J.O. Willis, D.E. MacLaughlin, C. Boekema, R.L. Lichti, J. Oostens, A.B. Denison, Phys. Rev. B 39, 11345 (1989).

[53] A. de Visser, R.J. Keizer, R. van Harrevelt, A.A. Menovsky, J.J.M. Franse, A. Amato, F.N. Gygax, M. Pinkpank, A. Schenck, Physica B 230-232, 53 (1997).

[54] R.J. Keizer, Ph.D. thesis, University of Amsterdam, 1999.

[55] Y. Kohori, M. Ishii, T. Kohara, J. Magn. Magn. Mater. 177-181, 441 (1998).

[56] P.H. Frings, private communication.

[57] P.H. Frings, B. Renker, C. Vettier, J. Magn. Magn. Mater. 63-64, 202 (1987).

[58] J.J.M. Franse, K. Kadowaki, A. Menovsky, M. van Sprang, A. de Visser, J. Appl. Phys. 61, 3380 (1987).

[59] S. Doniach, Physica B 91, 231 (1977).

[60] J.J.M. Franse, H.P. van der Meulen, A. de Visser, Physica B 165-166, 383 (1990).

[61] A. de Visser, R.J. Keizer, R. van Harrevelt, A.A. Menovsky, J.J.M. Franse, A. Amato, F.N. Gygax, M. Pinkpank, A. Schenck, J. Magn. Magn. Mater. 177-181, 435 (1998).

[62] A. de Visser, private communication. 
[63] A. Yaouanc, P. Dalmas de Réotier, G.N. Gygax, A. Schenck, A. Amato, C. Baines, P.C.M. Gubbens, C.T. Kaiser, A. de Visser, R.J. Keizer, A. Huxley, A.A. Menovsky, Phys. Rev. Lett. 84, 2702 (2000).

[64] R.J. Keizer, A. de Visser, A.A. Menovsky, J.J.M. Franse, A. Amato, F.N. Gygax, M. Pinkpank, A. Schenck, J. Phys., Condens. Matter 11, 8591 (1999).

[65] A. de Visser, M.J. Graf, P. Estrela, A. Amato, C. Baines, D. Andreica, F.N. Gygax, A. Schenck, Phys. Rev. Lett. 85, 3005 (2000).

[66] M.J. Graf, R.J. Keizer, A. de Visser, S.T. Hannahs, Physica B 284-288, 1281 (2000) 